\title{
Epstein-Barr Virus-Induced Polymorphic Nodal Lymphoproliferative Disorder Presenting as Recurrent Fever and Scrotal Swelling: A Case Report and Review of Literature
}

\author{
Krishna Joshi ${ }^{\mathrm{a}}$, Kevin Kuriakose ${ }^{\mathrm{a}}$, Rahul Ravilla ${ }^{\mathrm{a}}$, Yogita Rochlani ${ }^{\mathrm{a}}$, Anwar Rjoop ${ }^{\mathrm{b}}$, \\ Daisy Alapat $^{\mathrm{b}}$, AppalaNaidu Sasapu ${ }^{\mathrm{a}, \mathrm{c}}$
}

\begin{abstract}
Epstein-Barr virus (EBV) has been associated with the development of lymphoproliferative disorders (LPDs). Adults rarely develop systemic EBV-positive LPDs. We report a case of EBV-associated LPD presenting as recurrent scrotal swelling and fever for over nearly 30 years in an immunocompetent adult. A 64-year-old Caucasian male presented with generalized weakness, myalgia and fever associated with scrotal swelling and bilateral groin pain. He reported that these symptoms have recurred yearly for the past 30 years, lasting 1 - 2 days each. Systemic examination was normal except genital exam positive for scrotal swelling without tenderness and bilateral tender inguinal lymphadenopathy. Computed tomography (CT) scan of chest, abdomen and pelvis showed bilateral lymphadenopathy along external iliac chains and groins. Histopathological examination showed EBVassociated LPD and patient was started on rituximab. EBV is known to persist as a lifelong low-level infection in memory B cells of normal healthy carriers. Our patient's disease course over three decades is likely secondary to this property, in the setting of an intact immune system.
\end{abstract}

Keywords: Epstein-Barr virus; Lymphoproliferative disorder; Lymphadenopathy; Fever

\section{Introduction}

Epstein-Barr virus (EBV) is the first known oncogenic virus in humans, and has been associated with the development of

\footnotetext{
Manuscript accepted for publication July 06, 2016

aDepartment of Internal Medicine, University of Arkansas for Medical Sciences, Little Rock, AR, USA

bDepartment of Pathology, University of Arkansas for Medical Sciences, Little Rock, AR, USA

cCorresponding Author: AppalaNaidu Sasapu, University of Arkansas for Medical Sciences, 4301 W Markham St, Slot \#508, Little Rock, AR 72205, USA.Email: asasapu@uams.edu
}

doi: http://dx.doi.org/10.14740/jmc2579w various B-cell lymphoproliferative disorders (LPDs) such as Burkitt's lymphoma, classic Hodgkin's lymphoma, and lymphomas arising in immunocompromised individuals, even though EBV infection-associated lymphocytosis predominantly shows increased cytotoxic T cells. EBV-positive T-cell LPDs are commonly seen in children, especially in Asia. T-cell or natural killer (NK) cell lymphomas such as angioimmunoblastic T-cell lymphoma and extranodal nasal type NK/T-cell lymphoma show a strong association with chronic active EBV infection. Primary infection with EBV is generally asysmptomatic and occurs at a younger age. Adults rarely develop systemic EBV-positive LPDs [1]. We report a case of EBV-positive polymorphic nodal LPD presenting as recurrent scrotal swelling and fever over nearly 30 years.

\section{Case Report}

A 64-year-old Caucasian male presented to the emergency department with complaints of generalized weakness, myalgia and fever for a day. The fever was up to $102^{\circ} \mathrm{F}$, associated with fatigue, headache, scrotal swelling and pain at bilateral inguinal regions. He also reported mild productive cough for 2 days without chest pain or shortness of breath. History was negative for sore throat, runny nose, chills, night sweats, abdominal pain, dysuria or rash. He denied any penile discharge or genital lesions. He also reported a weight loss of about 7 lbs over the past few weeks, which he attributed to liraglutide that was started at about the same time. Review of past medical records revealed that he has been presenting to the hospital almost every year for past few years for similar complaints. Patient reported that he has been having these symptoms for the past 30 years. Each episode was associated with fever, myalgia, and scrotal swelling with pain at inguinal regions. He was treated with antibiotics each time without clear focus of infection. Antibiotics choices differed each time depending on the working diagnoses at the time, including possible pneumonia and tick borne illness. He would feel better over the next 1 or 2 days and would then be discharged. He would remain symptom free till his next yearly episode.

He has an extensive past medical history of atrial-flutter status post ablation, coronary artery disease, bradycardia with pacemaker placement, diabetes mellitus, psoriasis and obstruc- 


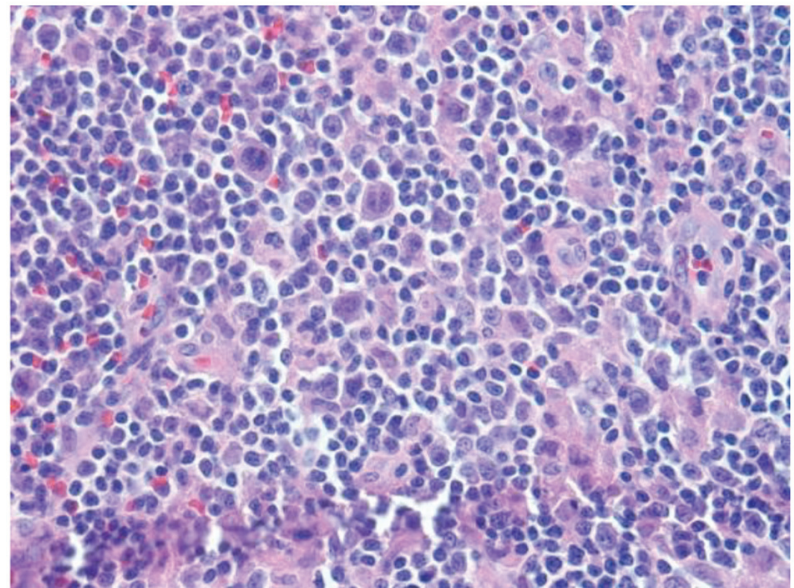

Figure 1. H\&E sections reveal lymph node with an altered architecture with vague nodularity, separated by fibrotic bands, and lymphoid infiltration into the perinodal adipose tissue. Nodules are composed of mixed inflammatory infiltrates consisting of polymorphous infiltrate comprising small lymphocytes, histiocytic aggregates, large atypical cells, immunoblasts, mummified cells, neutrophils/eosinophils and plasma cells, rare atypical RS type cells and increased vascularity comprised of high endothelial vessels.

tive sleep apnea. He denied family history of similar symptoms or autoimmune diseases. He reported no recent travel, tick bites or sick contacts. Physical exam showed blood pressure of $139 / 58 \mathrm{~mm} \mathrm{Hg}$, heart rate of $91 / \mathrm{min}$, temperature of $99.9^{\circ} \mathrm{F}$, and respiratory rate of $18 / \mathrm{min}$. Cardiac exam showed normal heart sounds and grade II-III systolic murmur best heard over the left sternal boarder. Abdominal, respiratory and central nervous system exams were within normal limits. Genital exam showed scrotal swelling without tenderness and bilateral small tender inguinal lymphadenopathy. No cervical or axillary lymphadenopathy was noted.

He was admitted to the hospital for evaluation of fever and weakness. CBC showed WBC of $15,600 / \mathrm{mm}^{3}$ with $\mathrm{Hb}$ of $15.2 \mathrm{~g} / \mathrm{dL}$ and platelets of $135,000 / \mathrm{mm}^{3}$. Urinalysis and chest $\mathrm{X}$-ray were normal. Peripheral smear showed leukocytosis

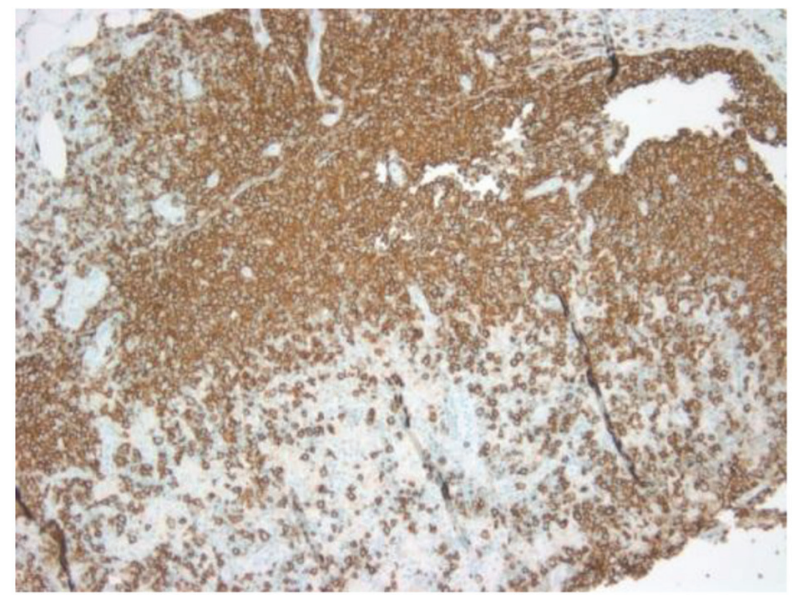

Figure 2. CD20 showing atypical large B cells.

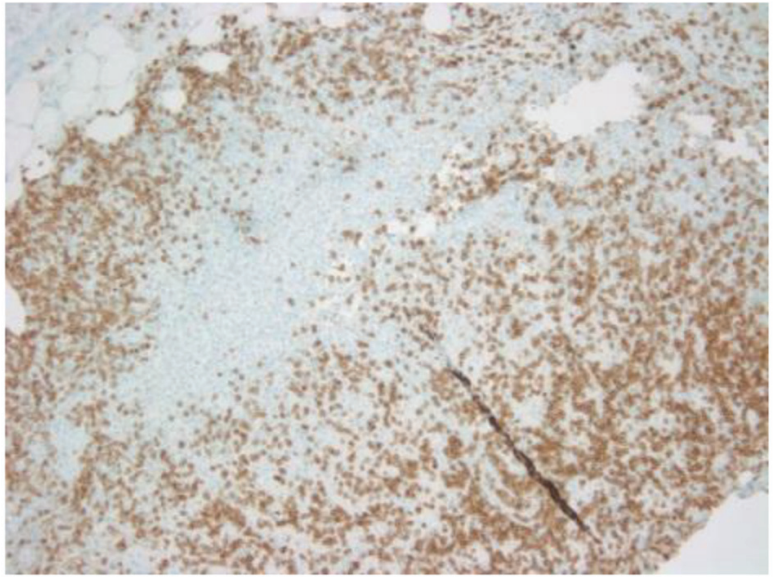

Figure 3. CD3 showing scattered positivity in atypical large T cells.

with predominantly mature, reactive neutrophils with lymphopenia and few reactive forms of lymphocytes without atypical cells. Mild thrombocytopenia was seen without morphologic abnormalities and no microorganisms or cellular inclusions were identified. Erythrocyte sedimentation rate was increased to 42 , C-reactive protein was increased to 186 , and creatine kinase was normal. He had mild hyponatremia with sodium of $132 \mathrm{mEq} / \mathrm{L}$ and mild hypokalemia with potassium of 3.4 $\mathrm{mEq} / \mathrm{L}$ with normal calcium, bicarbonate, chloride and renal functions. Broad spectrum antibiotics were started at admission and were discontinued after the cultures were negative and no source of infection was apparent. Anti-nuclear antibody titer and HIV tests were negative. Computed tomography (CT) scan of chest, abdomen and pelvis showed bilateral lymphadenopathy along the external iliac chains and groins without lymphadenopathy in the chest or abdomen. No splenomegaly was present.

Inguinal lymph node excisional biopsy was performed and sent for molecular analysis and histopathological examination. Molecular study for a clonal T-cell gene rearrangement was positive and was negative for a clonal B-cell gene rearrangement. Histopathological examination revealed a lymphnode

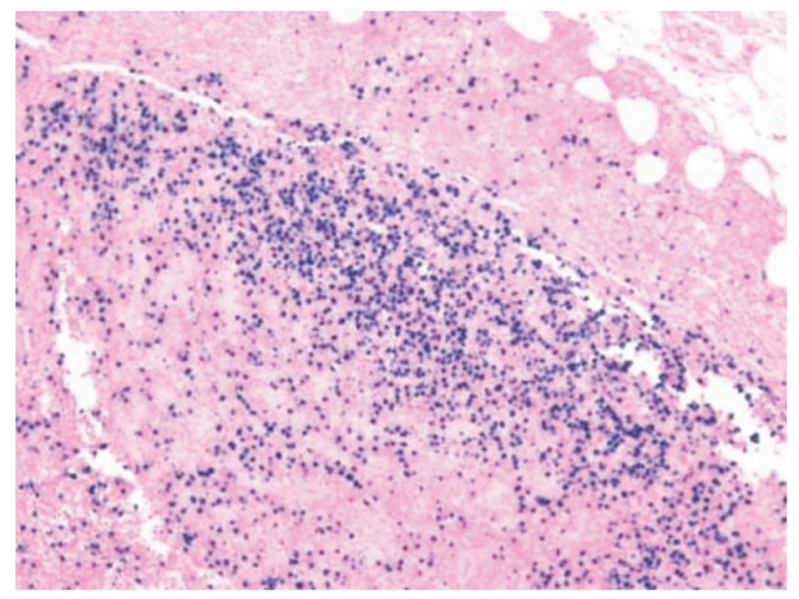

Figure 4. EBV in situ hybridization positive in B and T cells. 
with an atypical polymorphous inflammatory infiltrate, composed of large atypical lymphoid cells, Reed-Sternberg (RS) type cells, immunoblasts, mummified cells, histiocytic aggregates admixed with lymphocytes, neutrophils/eosinophils and plasma cells. Further immunohistochemical studies revealed that a large subset of the B and T lymphoid cells were positive for EBV encoded RNA by in situ hybridization (Figs. 1-4). The patient was thus diagnosed with EBV-associated polymorphic nodal LPD. Plasma EBV levels by quantitative PCR was not detected. Medical oncology was consulted for the management of EBV-associated LPD and the patient was started on rituximab.

\section{Discussion}

EBV-induced LPD usually presents with recurrent or persistent infectious mononucleosis-like symptoms incluidng fever, lymphadenopathy, and hepato-splenomegaly. EBV-associated LPD has been defined as EBV-positive B-cell LPD or EBVpositive T/NK-cell LPD depending on B- or T-cell positivity [2]. It can involve any organ but most commonly involves the lymph nodes, skin, liver, spleen, and bone marrow [3]. Our patient presented with fever and inguinal swelling on and off for about 30 years, feeling relatively well in between very short yearly symptomatic episodes. This was perhaps not picked up early since he would not always present to the same hospital each time, and sometimes simply waited at home for spontaneous resolution of symptoms. EBV infection is closely associated with certain LPDs. This disease mainly affects people of Asian origin and is uncommon in the western hemisphere [4]. EBV-positive nodal LPD is found to be more common in children but studies have shown that the adult cases have poorer prognoses and shorter survival times [5]. Our patient had these symptoms for several years and appeared to be stable and persistent, with a relapsing-remitting pattern. It thus remains unclear when he developed the LPD.

EBV mainly has affinity for B cells but can infect other cells as well. The mechanism by which it infects T and NK cell that do not express CD21 is unclear. It has been postulated that NK cells activated by EBV-infected B cells acquire CD21 by synaptic transfer which allow EBV binding to hosts $[6,7]$. The exact role of EBV in cell transformation is still being ascertained, and both viral and host factors may play a role. EBV has been shown to infect B lymphocytes in resting state and use its growth programme to drive the cells into activated proliferating lymphoblasts $[8,9]$. EBV-associated LPD usually occurs many years after the primary EBV infection, perhaps indicating the need for secondary transforming events. This disease can evolve into a more aggressive neoplasm or remain a benign or borderline condition with a high risk of evolution into a cytotoxic T-cell or NK-cell lymphoma. In our case, the patient's EBV-DNA in peripheral blood was negative but his biopsies were positive for EBV-associated LPD. Molecular studies for B-cell receptor gene rearrangement were negative but T-cell receptor gene rearrangement studies were positive (Figs. 1-3). Given our patient's localized lymphadenopathy with a T-cell monoclonality and vague B symptoms for sev- eral years, the findings were inferred to be most suggestive of EBV-associated polymorphic nodal LPD. EBV is known to persist as a lifelong low-level infection in memory B cells of normal healthy carriers $[6,10]$. Our patient's intact immune system may be responsible for the stable persistent relapsingremitting type of disease course over nearly three decades.

Monoclonal antibodies like rituximab have provided promising outcome in the targeted therapy of EBV-associated LPD [11]. EBV-positive LPDs are highly heterogeneous with regard to pathology and the host background against which the diseases arise. In general, chemotherapy is the mainstay of treatment in immunocompetent patients. Chemotherapy with regimen used in the treatment of lymphoma such as cyclophosphamide, docxorobucin, vincristine, prednisone (CHOP) remains a therapeutic option for patients who fail to respond to immune therapy or rituximab [12]. Our patient was started on treatment with rituximab, and his response will be closely monitored.

\section{References}

1. Rezk SA, Weiss LM. Epstein-Barr virus-associated lymphoproliferative disorders. Hum Pathol. 2007;38(9):12931304.

2. Cohen JI, Kimura H, Nakamura S, Ko YH, Jaffe ES. Epstein-Barr virus-associated lymphoproliferative disease in non-immunocompromised hosts: a status report and summary of an international meeting, 8-9 September 2008. Ann Oncol. 2009;20(9):1472-1482.

3. Quintanilla-Martinez L, Kimura H, Jaffe E. EBV-positive T-cell lymphoproliferative disorders of childhood. In: Swerdlow S, Campo E, Harris N, editors. International Agency for Research on Cancer. WHO classification of tumours of haematopoietic and lymphoid tissues. 4th edition. Geneva, Switzerland: World Health Organization; 2008. pp. 278-280.

4. Park S, Ko YH. Epstein-Barr virus-associated T/natural killer-cell lymphoproliferative disorders. J Dermatol. 2014;41(1):29-39.

5. Zheng XD, Zhou XG, Jin Y, Xie JL, Wei XJ, Chen SY, Mei X, et al. [Clinicopathologic features of systemic EBV-positive T/NK-cell lymphoproliferative disease in adults]. Zhonghua Bing Li Xue Za Zhi. 2011;40(4):227234.

6. Thorley-Lawson DA, Allday MJ. The curious case of the tumour virus: 50 years of Burkitt's lymphoma. Nat Rev Microbiol. 2008;6(12):913-924.

7. Su IJ, Lin KH, Chen CJ, Tien HF, Hsieh HC, Lin DT, Chen JY. Epstein-Barr virus-associated peripheral Tcell lymphoma of activated CD8 phenotype. Cancer. 1990;66(12):2557-2562.

8. Carbone A, Gloghini A, Dotti G. EBV-associated lymphoproliferative disorders: classification and treatment. Oncologist. 2008;13(5):577-585.

9. Aman P, Ehlin-Henriksson B, Klein G. Epstein-Barr virus susceptibility of normal human B lymphocyte populations. J Exp Med. 1984;159(1):208-220.

10. Thorley-Lawson D . EBV persistence and latent infection 
in vivo. ES R, editor. Epstein-Barr Virus. 1st ed. Norfolk, England: Caister Academic Press. pp. 309-349.

11. Thorley-Lawson DA, Mann KP. Early events in EpsteinBarr virus infection provide a model for B cell activation. J Exp Med. 1985;162(1):45-59.
12. Habermann TM, Weller EA, Morrison VA, Gascoyne RD, Cassileth PA, Cohn JB, Dakhil SR, et al. Rituximab$\mathrm{CHOP}$ versus $\mathrm{CHOP}$ alone or with maintenance rituximab in older patients with diffuse large B-cell lymphoma. J Clin Oncol. 2006;24(19):3121-3127. 